\title{
The evolving role of the renin-angiotensin system in ARDS
}

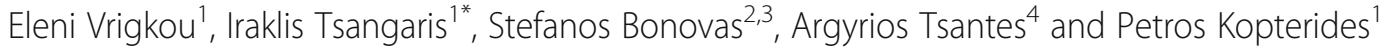 \\ See related research by Khan et al., https://ccforum.biomedcentral.com/articles/10.1186/s13054-017-1823-x
}

We read with great interest the article by Khan et al. [1] reporting the results of a pilot clinical trial of administrating recombinant angiotensin-converting enzyme 2 (ACE2) in patients with acute respiratory distress syndrome (ARDS). Current research has been focused on the involvement of the renin-angiotensin system (RAS) in the pathogenesis and clinical outcomes of ARDS and, in particular, in the evaluation of the angiotensin-converting enzyme (ACE)/ angiotensin II (Ang II)/Ang II receptor type 1 axis and its counterpart ACE2/angiotensin-(1-7)/MAS1 receptor pathway as potential therapeutic targets. Given the lack of effective pharmacological ARDS treatments, clinical trials exploring new therapeutic strategies, like that by Khan et al. [1], are essential.

The association of ACE activity with ARDS outcomes is far from linear. Multiple factors, like the heterogeneity of ARDS and the variability of RAS activation in patient subgroups, could be implicated in this complex interplay [1]. Even though increased levels of ACE and Ang II have been detected in patients with ARDS, the pathophysiological role of RAS in ARDS development remains unclear. Additional factors such as, for example, the variable substrates of ACE and ACE2 apart from Ang (namely bradykinin and apelin), the protective effects against lung injury which Ang II receptor type 2 exerts, and the effect of ACE2 via the MAS1 receptor (and not via the Ang II receptor type 1) could be implicated [2]. There are encouraging data from animal models on the positive effects of ACE2 in ARDS, but respective data involving humans are insufficient [2].

In our study [3], higher ACE levels were not associated with the oxygenation index, even though they (but not the D/D genotype, which relates to higher serum ACE levels) seemed to correlate with ARDS prognosis. Zambelli et al. [4] showed improved arterial oxygenation (although not clinically relevant) in an experimental ARDS model after administration of angiotensin-(1-7), but no difference in survival or pulmonary mechanics. In Khan et al.'s study [1], administration of recombinant ACE2 in ARDS patients decreased Ang II levels without causing hypotension, but worsened respiratory mechanics. Khanna et al. [5] demonstrated in ATHOS-3 that Ang II administration in patients with vasodilatory shock increased blood pressure, without raising the risk for ARDS.

It is apparent that there are variable and often conflicting data in the current literature on the complex association between RAS and ARDS, which warrant the conduction of additional clinical trials to further elucidate its pathophysiological background and evaluate potential new treatments.

\section{Authors' response}

\section{Andrew I. Bayliffe, Akram Khan and Aili L. Lazaar}

We are grateful for the comments of Vrigkou et al., and share their views regarding the need for more investigations into RAS biology in ARDS and other acutely ill patients. In addition to the well-documented effects on vascular tone

\footnotetext{
* Correspondence: itsagkaris@med.uoa.gr

${ }^{1}$ 2nd Department of Critical Care Medicine, Attikon University Hospital, National and Kapodistrian University of Athens, Rimini Str. 1, Athens 12462, Greece

Full list of author information is available at the end of the article
}

and fluid/electrolyte homeostasis, the "classical" RAS system, through the action of Ang II and its receptor angiotensin receptor 1 (AT1R), elicits inflammation, oxidative stress, cellular apoptosis, and fibroproliferative responses [6], all important components of ARDS pathophysiology. ACE2 has been shown to improve both pathophysiology and outcomes in various animal models of ARDS by reducing Ang II/AT1R signaling and activating the regulatory angiotensin-(1-7)/Mas1 pathway, as 
demonstrated by studies that administer ACE2 or angiotensin-(1-7) peptide alone $[7,8]$.

In relation to the genetic evidence supporting a role for the RAS in human ARDS, we agree with Vrigkou et al. that the data are somewhat contradictory, probably because heterogeneity within ARDS cohorts hampers interpretation of genetic association studies. Despite this limitation, there are data from meta-analyses suggesting an association of the D-allele of the $A C E$ gene with both ARDS risk [9] and mortality [3]. Together with work elucidating ACE2 as the receptor for the severe acute respiratory syndrome (SARS) coronavirus that caused uncharacteristically severe lung injury associated with profound depletion of ACE2 [10], these data suggest an important role for ACE2 in ARDS pathophysiology.

Our pilot clinical trial was designed to establish preliminary safety and tolerability, with an emphasis on monitoring hemodynamic stability, and to define exposure-response relationships with the RAS system. As mentioned in our discussion, readers should exercise caution when interpreting the "effects" of study treatments on physiology and respiratory mechanics given the small sample size and the risk of ascertainment bias at later time points, where only a small number of patients contribute to point estimates and significant imbalances between groups are apparent.

Finally, the contrast with the ATHOS-3 trial [5] where Ang II infusions elicited acute improvements in MAP in patients with high-output shock is important. Rather than revealing a novel protective role of Ang II via its other receptor AT2R, we believe this confirms the well-established vasopressor effects of Ang II, mediated via AT1R. To rationalise whether classical RAS signaling is beneficial or harmful in acutely ill patients, more trials with Ang II and ACE2 are required to fully understand their longer-term safety profile, their impact on RAS and other biological systems, and their impact on organ failure and outcomes. In the meantime, we speculate that there may be a place for both therapies in different patient populations, with short-term repletion of Ang II in certain vasoplegic shock patients with low Ang II levels, and repletion of ACE2 in ARDS patients with a high Ang II/angiotensin-(1-7) ratio.

\section{Abbreviations}

ACE: Angiotensin-converting enzyme; ACE2: Angiotensin-converting enzyme 2; Ang II: Angiotensin II; ARDS: Acute respiratory distress syndrome;

RAS: Renin-angiotensin system

\section{Acknowledgements}

None to report.

Funding

No funding to report.

Availability of data and materials

Not applicable.

\section{Authors' contributions}

EV, IT, SB, AT, and PK contributed to the concept, design, and writing of the manuscript. All authors read and approved the final manuscript.

Ethics approval and consent to participate

Not applicable.

Consent for publication

Not applicable.

Competing interests

The authors declare that they have no competing interests.

\section{Publisher's Note}

Springer Nature remains neutral with regard to jurisdictional claims in published maps and institutional affiliations.

\section{Author details}

'2nd Department of Critical Care Medicine, Attikon University Hospital, National and Kapodistrian University of Athens, Rimini Str. 1, Athens 12462, Greece. ${ }^{2}$ Department of Biomedical Sciences, Humanitas University, Milan, Italy. ${ }^{3}$ Humanitas Clinical and Research Center, Milan, Italy. ${ }^{4}$ Laboratory of Hematology and Blood Bank Unit, Attikon University Hospital, Athens, Greece.

Received: 7 November 2017 Accepted: 4 December 2017

Published online: 28 December 2017

\section{References}

1. Khan A, Benthin C, Zeno B, et al. A pilot clinical trial of recombinant human angiotensin-converting enzyme 2 in acute respiratory distress syndrome. Crit Care. 2017;21:234.

2. Imai $Y$, Kuba $K$, Penninger JM. Angiotensin-converting enzyme 2 in acute respiratory distress syndrome. Cell Mol Life Sci. 2007;64:2006-12.

3. Tsantes $A E$, Kopterides $P$, Bonovas $S$, et al. Effect of angiotensin converting enzyme gene I/D polymorphism and its expression on clinical outcome in acute respiratory distress syndrome. Minerva Anestesiol. 2013;79:861-70.

4. Zambelli V, Bellani G, Borsa R, et al. Angiotensin-(1-7) improves oxygenation, while reducing cellular infiltrate and fibrosis in experimental Acute Respiratory Distress Syndrome. Intensive Care Med Exp. 2015;3:8.

5. Khanna A, English SW, Wang XS, et al. Angiotensin II for the treatment of vasodilatory shock. N Engl J Med. 2017:377:419-30.

6. Oudit GY, Crackower MA, Backx PH, Penninger JM. The role of ACE2 in cardiovascular physiology. Trends Cardiovasc Med. 2003:13:93-101.

7. Zou Z, Yan Y, Shu Y, Gao R, Sun Y, Li X, Ju X, Liang Z, Liu Q, Zhao Y, et al. Angiotensin-converting enzyme 2 protects from lethal avian influenza $A$ H5N1 infections. Nat Commun. 2014;5:3594.

8. Klein N, Gembardt F, Supe $S$, et al. Angiotensin-(1-7) protects from experimental acute lung injury. Crit Care Med. 2013;41:334-43.

9. Deng $X$, Zhang $S$, Jin $K$, et al. Angiotensin-converting enzyme I/D polymorphism and acute respiratory distress syndrome. J RAAS. 2015;16(4): 780-6.

10. Kuba K, Imai $Y$, Rao $S$, et al. A crucial role of angiotensin converting enzyme 2 (ACE2) in SARS coronavirus-induced lung injury. Nat Med. 2005;11:875-9. 